\title{
An Investigation on Wireless Mobile Network and Wireless LAN (Wi-Fi) for Performance Evaluation
}

\author{
Rakesh Kumar Singh \\ Scientist-D (IT) \\ G.B. Pant Institute of Himalayan Environment \& \\ Development, Kosi-Katarmal, Almora, \\ Uttarakhand, India and \\ Ph.D. Scholar, Kumaun University, Nainital
}

\author{
Neeraj Tiwari \\ Professor \& Head \\ Department of Statistics, \\ Kumaun University, SSJ Campus, \\ Almora, Uttarakhand, India
}

\begin{abstract}
The convergence of wireless mobile technologies and Internet allows compelling possibilities for future applications due to the new advancement, constantly growing and personalized services in the mobile communication. 3G (third generation) and $\mathrm{Wi}-\mathrm{Fi}$ are major wireless technologies that are used by most people today. The two most important phenomena impacting telecommunications over the past decade have been explosive parallel growth of both the internet and mobile telephone services. The internet brought the benefits of data communications to the masses with email, the web, and ecommerce; while mobile service has enabled "follow-me anywhere/always on" telephony. The Internet helped accelerate the trend from voice centric to data-centric networking. The scope of this research is to carry out extensive study with respect to two wireless technologies that are likely to play important roles: Third Generation mobile (3G) wireless mobile network and Wireless Local Area Networks (Wi-Fi). The performance will be evaluated in different operating environment with the use of various analytical techniques. Focus will be given on $3 \mathrm{G}$ wireless mobile network as embodied by the IMT-2000 family of standards and Wireless LAN technology embodied by the Wi$\mathrm{Fi}$ or $802.11 \mathrm{~b}$ standard which is the most popular and widely deployed of the Wireless LAN technologies.
\end{abstract}

\section{Keywords}

Wireless Mobile Network, 3G, Wi-Fi, Wireless Network, WLAN, UMTS, etc.

\section{INTRODUCTION}

Wireless communications are becoming ubiquitous in homes, offices and enterprises with the ability to provide high-speed, high-quality information exchange between portable devices such as computers, mobile phones, tablets, PDAs, IP phones, etc. It brings great benefit in areas like temporary installations in buildings where wiring is logistically difficult, or in locations such as college campuses or airports where users are not likely to do all of their computing in one fixed spot. With the advancement of modern wireless technology, most people prefer to avoid Ethernet cables to connect to computers. Nowadays, even certain emergency services are transferring their confidential data through a wireless network. Using wireless network interfaces, mobile devices can be connected to the public telephone network in the same way as wired telecommunications, or to the Internet in the same way as desktop computers are connected, using the Ethernet, a token ring, or point-to-point links. With the tremendous success of wireless telephony and messaging services, it is hardly surprising that mobile Internet access services (e.g., via cellular/mobile networks such as GPRS, UMTS, WLAN-WiFi) which provide Internet access "anywhere" and "any time" are likely to experience dramatic growth. Nowadays, there are three main wireless access technologies: Bluetooth, WLAN (Wi-Fi) 802.11 and 3G Wireless networks (e.g., GSM/GPRS, UMTS). 3G Wireless networks provides high ubiquity and mobility to customers and mobile cellular technologies are evolving from GPRS to UMTS. WLAN (Wi-Fi) hotspots or networks are still spreading rapidly. Wi-Fi technologies are currently capable of providing high capacity wireless LAN coverage within a limited area.

\section{OBJECTIVES OF THE RESEARCH}

The broad objectives of the research are as follows:

- A comparative study for services and applications of $3 \mathrm{G}$ wireless mobile network offered by various telecommunication operators in India.

- Statistical analysis of $3 \mathrm{G}$ wireless mobile network \& Wi-Fi network data collected through primary and secondary resources.

- Study on Wireless LAN which will be focused only on Wi-Fi network and its comparison with 3G wireless mobile network.

- Performance evaluation for 3G wireless mobile network and $\mathrm{Wi}-\mathrm{Fi}$ network in different operating environment through various analytical techniques to identify success trends in India.

- Pilot study for 4G wireless mobile network and its comparison with 3G wireless mobile network (elective objective)

\section{RESEARCH QUESTIONS}

The research questions have intended to exercise the user defined node models over a range of normal operating conditions and demonstrate the functionality of the simulation environment. The following research questions will be addressed in this research:

- What is the societal impact of 3G wireless mobile network as compared to traditional wired based network?

- What is the adoption rate for the services and applications of $3 \mathrm{G}$ wireless mobile network in India?

- What is the societal impact and future of Wireless LAN (Wi-Fi)?

- What is the performance of $3 \mathrm{G}$ wireless mobile network and Wi-Fi under different operating environment? 


\section{HYPOTHESIS}

A hypothesis is a prediction of expected outcomes, it states the relationships between variables that the researcher expects to find as a result of the study or in other words a hypothesis is an educated guess or proposition that attempts to explain a set of facts or natural phenomenon. The following hypothesis will be considered in this research:

- IP based 3G wireless mobile network and Wi-Fi is better than non IP based wireless network.

- $\quad 3 \mathrm{G}$ wireless mobile network and Wi-Fi network perform better while applications are running individually rather than simultaneously.

- Performance of wireless mobile network is better while using IPv4 than IPv6.

- Geographical coverage area of 3G wireless mobile network is larger than Wi-Fi network.

\section{METHODOLOGY}

Research methodology defines the research activity, development of research activity and measurements used to advance the research work by implementing these measures which assist to achieve the objectives of the research. Quantitative and qualitative research methodologies will be used in this research because this study is about survey on various services and applications of $3 \mathrm{G}$ wireless mobile network and Wi-Fi among different user groups and telecommunication operators. The network performance and comparison between $3 \mathrm{G}$ wireless mobile network and Wi-Fi in different operating environment will be carried out for ensuring quality of service. The broad methodology is as follows:

- Approach: This is the beginning phase that includes comprehensive literature survey for better and clean understanding of the research work done in the past on $3 \mathrm{G}$ wireless mobile network and Wi-Fi. The basic 3G \& Wi-Fi principles and methods will be illustrated based on the available literature studies.

- Data Collection: This phase consists of the steps which lead to survey and collection of primary and secondary data through various sources.

- Data Analysis: After the collection of data is done, the observations and findings are then analyzed with the help of various statistical methods and tools.

- Comparative Analysis: This phase consists study on Wi-Fi network and its comparison with $3 \mathrm{G}$ wireless mobile networks on various wireless network parameters.

- Performance Evaluation: Performance evaluation will be carried out in different operating environment for $3 \mathrm{G}$ wireless mobile network and Wi-Fi network.

- Communication of Research: Summary, discussions, findings and recommendations will be completed in this phase.

- Thesis: Final thesis will be prepared based on research work.

\section{REVIEW OF LITERATURE}

The literature review provides a framework for establishing the importance of the study. Extensive literature review has been carried by visiting the libraries of various Universities, Institutes, organizations and by exploring secondary information which is available on public domain/Internet. It has been found that number of research on $3 \mathrm{G}$ wireless mobile network and Wi-Fi have been carried out in other countries in past but in context of India, very less research work has been done on $3 \mathrm{G}$ wireless mobile network and Wi-Fi.

Ahonen and Barrett (2002) provide information about the heart of the UMTS (Universal Mobile Telecommunication Services or System) innovation will be the terminal and a new way of using the mobile phone. The mobile subscribers now want to more personalization and entertainment in the phone rather than the voice. The strong growth in mobile voice will continue in mobile data. The UMTS terminal will become a service platform which capable of multiple radio access modes and compliant with open standards and operating systems to enable mobile internet and mobile multimedia messaging services.

Balachandran et al. (2002) analyzed user behavior and network performance in public area wireless networks. They developed a parameterized model for wireless users for use with analytic and simulation studies and for the application of workload analysis results to issues in wireless network deployment such as capacity planning and potential network optimizations.

Robins et al. (2003) discussed current features of mobile communication technologies. Since $20013 \mathrm{G}$ trials across the world have shown that the main development from $2 \mathrm{G}$ and $2.5 \mathrm{G}$ to $3 \mathrm{G}$ is faster connection speed referring to wider bandwidth. The main characteristics of $3 \mathrm{G}$ are to provide mobile multimedia services at a transmission rate of $144 \mathrm{kbps}$ at the high speed, $384 \mathrm{kbps}$ at the speed of walking and $2 \mathrm{Mbps}$ indoors. This theoretical maximum (2Mbps) is close to the speed of LAN connections that many households nowadays have.

\section{WHAT IS WIRELESS NETWORK?}

A wireless network is any type of computer network that uses radio or micro waves to connect network devices such as mobile phones, Wi-Fi gadgets, laptops, tablets, etc. Wireless networking is a method by which homes, telecommunications networks and enterprise installations avoid the costly process of introducing cables into a building or as a connection between various equipment locations. Wireless telecommunications networks are generally implemented and administered using radio communication. This implementation takes place at the physical layer of the OSI reference model structure. Examples of wireless networks include cell phone network or wireless mobile network, wireless LAN (Wi-Fi) and terrestrial microwave networks.
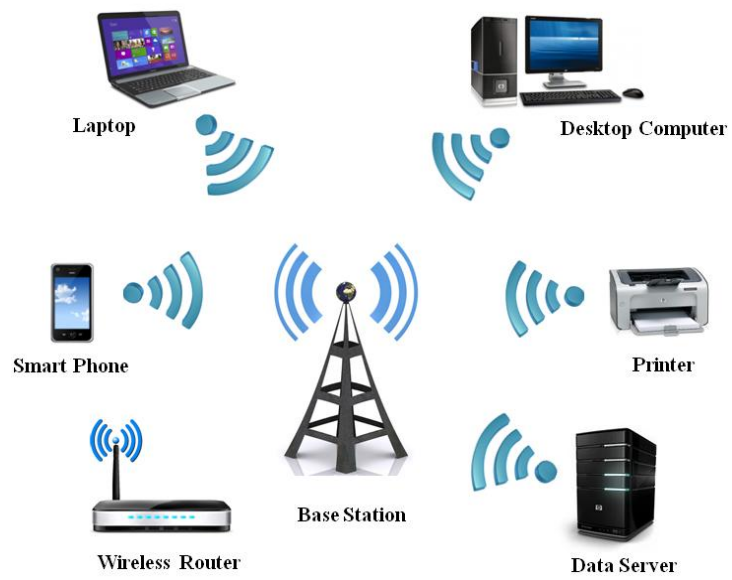

Fig.1: Typical structure of wireless network. 


\subsection{Significances of Wireless Network}

Presently, wireless network is becoming more and more popular for accessing various data and voice services over the wireless devices. The following are the few benefits of the wireless network:

- Convenience: Easy access of network resources from any location within wireless network's coverage area or from any Wi-Fi hotspot.

- Easy Setup: Installation of wireless network is very less time taking and cost-effective because of non requirement of string cables and wires.

- Expandable: Wireless networks can easily be expanded with existing equipment while a wired network might require additional wiring and network equipments.

- Security: Advances in wireless networks provide robust security protections.

- Mobility: Wireless devices can be moved from one location to another within the geographical coverage area of wireless network for accessing network services without any interruption.

- Productivity: Wireless availability of particular application or internet to access resource of the business can increase the productivity of the staff and the business organisation.

- Cost Effective: Wireless networks can cost less to operate than wired networks because it eliminate or reduce wiring and equipment costs.

\section{WIRELESS MOBILE NETWORK}

A wireless mobile network or cellular network is a wireless radio network distributed over land areas called cells. Each cell served by at least one fixed-location transceiver known as a cell site or base station. In a wireless mobile network each cell characteristically uses a different set of radio frequencies from all their immediate neighbouring cells to avoid any interference and provide guaranteed bandwidth within each cell. When joined together these cells provide radio coverage over a wide geographic area. This enables a large number of portable transceivers (e.g., mobile phones, laptops, tablets, etc.) to communicate with each other and with fixed transceivers and telephones anywhere in the network via base stations, even if some of the transceivers are moving through more than one cell during transmission.

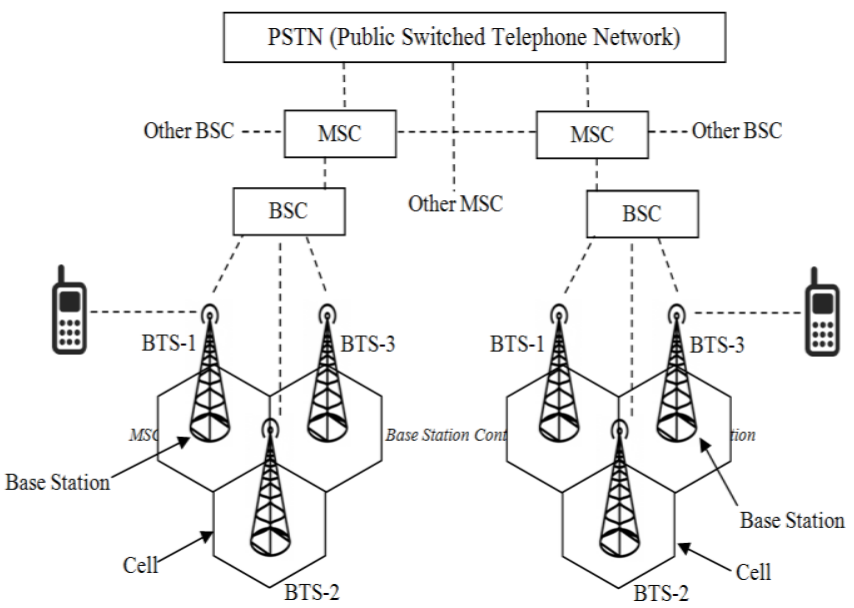

Fig.2: Basic architecture of cellular network or wireless mobile network.
Wireless mobile network offers a number of desirable characteristics:

- More capacity than a single large transmitter since the same frequency can be used for multiple links as long as they are in different cells.

- Mobile devices use less power than with a single transmitter or satellite since the cell towers are closer.

- Larger coverage area than a single terrestrial transmitter since additional cell towers can be added indefinitely and are not limited by the horizon.

\subsection{Mobile or Cellular Network Generations}

Wireless mobile network or cellular network generations are given as follows with respect to technological advancements in telecommunication technology:

- OG: Briefcase-size mobile radio telephones

- 1G: Analog cellular telephony

- 2G: Digital cellular telephony

- 3G: High-speed digital cellular telephony (including data and video telephony)

- 4G: IP-based "anytime and anywhere" voice, data and multimedia telephony at faster data rates than 3G (presently not deployed in India)

Major telecommunications providers have deployed voice and data wireless mobile network over most of the inhabited land area of the Earth. This allows mobile phones and mobile computing devices to be connected to the public switched telephone network (PSTN) and public Internet. Although originally intended for cell phones, with the development of smartphones, cellular telephone networks routinely carry data in addition to telephone conversations.

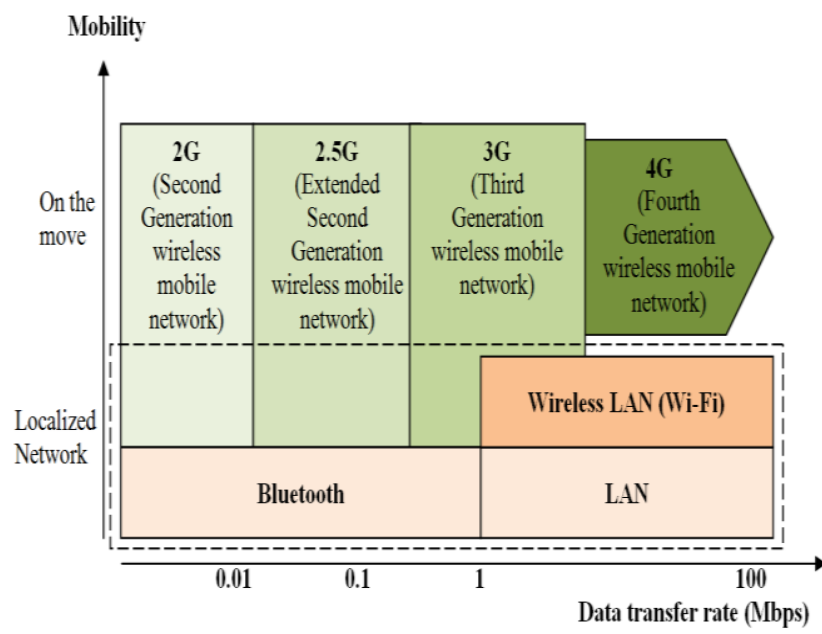

Fig.3: Evolution and classification of Wireless Mobile Network.

2G (Second Generation wireless mobile network) first introduced in 1992, is the second generation of cellular telephone technology and the first to use digital encryption of conversations. $2 \mathrm{G}$ networks were the first to offer data services and SMS text messaging but their data transfer rates are lower than those of their successors. The capabilities of 2G network are GSM 900 and GSM 1800. 
3G (Third Generation wireless mobile network) networks succeed $2 \mathrm{G}$ ones, offering faster data transfer rates and are the first to enable video calls. This makes them especially suitable for use in modern smart phones which require constant highspeed internet connection for many of their applications. The capabilities of 3G network is UMTS 2100.

4G (Fourth Generation wireless mobile network) is the fourth generation of mobile phone communications standards. It is a successor of the $3 \mathrm{G}$ and provides ultra-broadband internet access for mobile devices. The high data transfer rates make 4G networks suitable for use in USB wireless modems for laptops and even home internet access. The capabilities of $4 \mathrm{G}$ network is LTE 2300.

\section{THIRD GENERATION (3G) \\ WIRELESS MOBILE NETWORK}

$3 \mathrm{G}$ is the third generation of mobile telecommunications technology and is based on a set of standards used for mobile devices and mobile telecommunications use services and networks that comply with the International Mobile Telecommunications - 2000 (IMT-2000) specifications by the International Telecommunication Union (ITU). $3 \mathrm{G}$ finds application in wireless voice telephony, mobile Internet access, fixed wireless Internet access, video calls, mobile TV and other data and voice services. 3G telecommunication networks support services that provide a data transfer rate of at least $200 \mathrm{kbit} / \mathrm{s}$. Later $3 \mathrm{G}$ releases often denoted $3.5 \mathrm{G}$ and $3.75 \mathrm{G}$ also provide mobile broadband access of several Mbit/s to smart phones and mobile modems in laptop computers. Several telecommunications companies market wireless mobile Internet services as $3 \mathrm{G}$, indicating that the advertised service is provided over a $3 \mathrm{G}$ wireless network. Services advertised as $3 \mathrm{G}$ are required to meet IMT-2000 technical standards including standards for reliability and data transfer rates. A new generation of cellular standards has appeared approximately every tenth year since $1 \mathrm{G}$ systems were introduced in 1981-1982. Each generation is characterized by new frequency bands, higher data rates and non-backwardcompatible transmission technology. The first $3 \mathrm{G}$ network was introduced in 1998 .

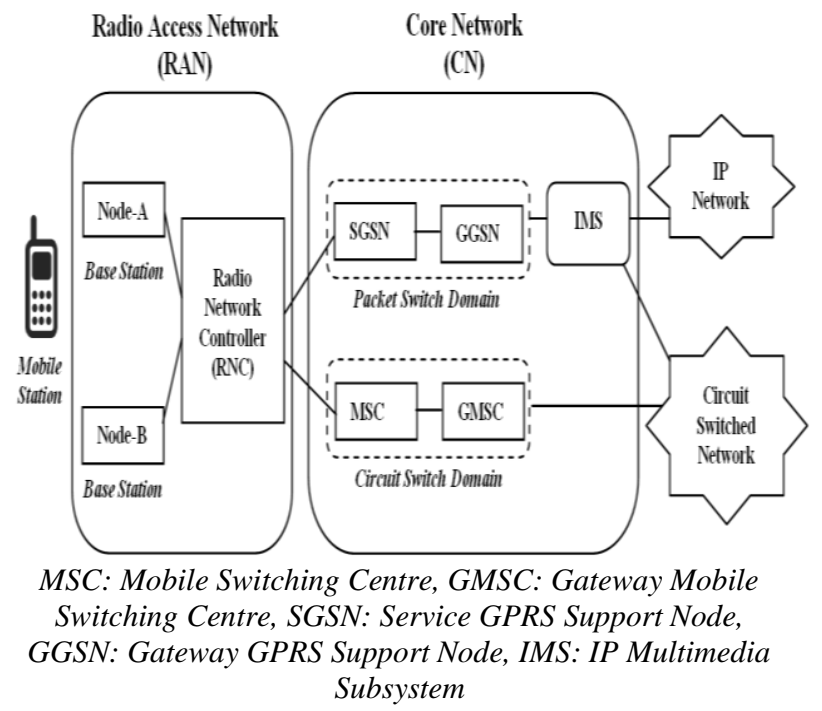

Fig.4: Basic architecture of 3G wireless mobile network.

\subsection{Third generation (3G) standards}

The following standards are typically branded $3 \mathrm{G}$ wireless mobile network:
- The Universal Mobile Telecommunications System (UMTS) created and revised by the 3GPP. The family is a full revision from GSM in terms of encoding methods and hardware, although some GSM sites can be retrofitted to broadcast in the UMTS/W-CDMA format UMTS system first offered in 2001 standardized by 3GPP. The mobile phones are typically UMTS and GSM hybrids. Several radio interfaces are offered sharing the same infrastructure:

- The latest UMTS release, HSPA+ which can provide peak data rates up to $56 \mathrm{Mbit} / \mathrm{s}$ in the downlink in theory $(28 \mathrm{Mbit} / \mathrm{s}$ in existing services) and $22 \mathrm{Mbit} / \mathrm{s}$ in the uplink.

- W-CDMA is the most common deployment, commonly operated on the $2,100 \mathrm{MHz}$ band. A few others use the 850,900 and $1,900 \mathrm{MHz}$ bands.

- HSPA is an amalgamation of several upgrades to the original W-CDMA standard and offers speeds of $14.4 \mathrm{Mbit} / \mathrm{s}$ down and $5.76 \mathrm{MBit} / \mathrm{s}$ up. HSPA is backward-compatible with and uses the same frequencies as W-CDMA.

- HSPA+, a further revision and upgrade of HSPA, can provide theoretical peak data rates up to $168 \mathrm{Mbit} / \mathrm{s}$ in the downlink and $22 \mathrm{Mbit} / \mathrm{s}$ in the uplink, using a combination of air interface improvements as well as multicarrier HSPA and MIMO. Technically though, MIMO and DC-HSPA can be used without the "+" enhancements of HSPA+

- CDMA2000 system first offered in 2002 standardized by $3 \mathrm{GPP} 2$, sharing infrastructure with the IS-95 2G standard. The mobile phones are typically CDMA2000 and IS-95 hybrids. The latest release EVDO Rev B offers peak rates of 14.7 Mbit/s downstream. The CDMA2000 system, or IS2000, including CDMA2000 1x and CDMA2000 High Rate Packet Data standardized by 3GPP2 (differing from the 3GPP), evolving from the original IS-95 CDMA system.

CDMA2000 1x Rev. E has an increased voice capacity (in excess of three times) compared to Rev. 0 EVDO Rev. B offers downstream peak rates of $14.7 \mathrm{Mbit} / \mathrm{s}$ while Rev. C enhanced existing and new terminal user experience.

\subsection{Basically $3 G$ is designed to deliver}

- High speed e-mail and Internet access

- A wide range of market focused applications

- Long term market driven creativity, an innovative value chain and real user benefits, driving genuine market demand

- Advanced, lightweight, easy-to-use terminals with intuitive interfaces

- Instant real-time multimedia communications

- Global mobility and roaming

- A wide range of vendors and operators offering choice, competition and affordability

- Applications of $3 \mathrm{G}$ are:

- High speed voice and data services

- Global Positioning System (GPS)

- Location-based services 
- Mobile TV

- Mobile Telephony

- Telemedicine

- Video Conferencing/Calling

- Video on demand

- Mobile Internet, browsing the web from mobile

- E-mail services

- Messaging services - short message service

(SMS) and multimedia message service (MMS)

- Mobile e-commerce

- Online gaming

\section{WIRELESS LAN (Wi-Fi)}

A wireless local area network (WLAN) or Wi-Fi is a wireless computer network that links two or more devices using a wireless distribution method (often spread-spectrum or OFDM radio) within a limited area such as a home, school, computer laboratory or office building. This gives users the ability to move around within a local coverage area and still be connected to the network and can provide a connection to the wider Internet. Most modern WLANs are based on IEEE 802.11 standards, marketed under the Wi-Fi brand name.

The wireless LAN (Wi-Fi) works in three basic configurations: peer-to-peer, bridge and wireless distribution system. Peer-to-peer configuration is where each computer in the network can act as a client or server for the other computers in the network. This allows them shared access to files (such as audio, video, data, etc.) and peripherals without needing a central server. A bridge configuration is used to connect networks. This is done by use of a wireless Ethernet bridge providing the connection for devices to a wireless network. The wireless distribution system enables the wireless inter-connection of the access points within a network. This allows a wireless network to be expanded through the use of multiple access points linked together. Generally, a Wi-Fi signal can reach up to 500 feet indoors and approximately 1000 feet outdoors. Wi-Fi operates in the unlicensed $2.4 \mathrm{GHz}$ or $5.8 \mathrm{GHz}$ ISM Band. Wi-Fi transmissions are essentially FM transmission, in that the frequency is changed to transmit data. Typically, the WLANs are implemented as part of a private network

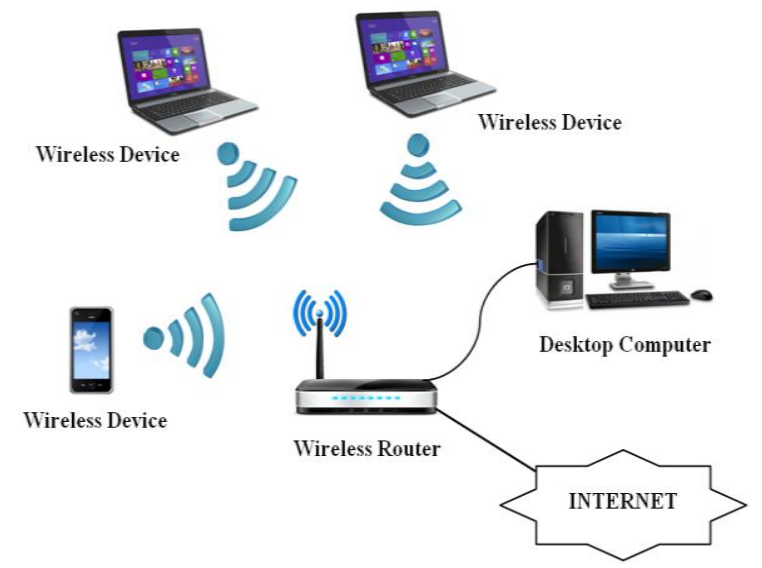

Fig.5: Basic architecture of wireless LAN (Wi-Fi).

\subsection{Architectural components of wireless LAN (Wi-Fi)}

The main architectural components of a wireless network are the wireless router (access point), Wi-Fi cards, safeguards and wireless clients. The typical components of wireless LAN (Wi-Fi) are as follows:
- Stations: All components that can connect into a wireless medium in a network are referred to as stations. All stations are equipped with wireless network interface controllers (WNICs). Wireless stations fall into one of two categories: wireless access points and clients. Access points (APs), normally wireless routers, are base stations for the wireless network. Wireless clients can be mobile devices such as laptops, personal digital assistants, IP phones and other smartphones, or fixed devices such as desktops and workstations that are equipped with a wireless network interface.

- Basic Service Set: The basic service set (BSS) is a set of all stations that can communicate with each other. Every BSS has an identification (ID) called the BSSID, which is the MAC address of the access point servicing the BSS. There are two types of BSS: Independent BSS (also referred to as IBSS) and infrastructure BSS. An independent BSS (IBSS) is an ad hoc network that contains no access points, which means they cannot connect to any other basic service set.

- Extended Service Set: An extended service set (ESS) is a set of connected BSSs. Access points in an ESS are connected by a distribution system. Each ESS has an ID called the SSID which is a 32-byte (maximum) character string.

- Distribution System: A distribution system (DS) connects access points in an extended service set. The concept of a DS can be used to increase network coverage through roaming between cells. DS can be wired or wireless. Current wireless distribution systems are mostly based on WDS or MESH protocols, though other systems are in use.

\subsection{Wi-Fi IEEE Standards}

The 802.11 standard is defined through several specifications of WLAN (Wi-Fi). It defines an over-the-air interface between a wireless client and a base station or between two wireless clients. There are several specifications in the 802.11 family:

- 802.11: This pertains to wireless LANs and provides 1 or 2 Mbps transmission in the $2.4-\mathrm{GHz}$ band using either frequency-hopping spread spectrum (FHSS) or direct-sequence spread spectrum (DSSS).

- 802.11a: This is an extension to 802.11 that pertains to wireless LANs and goes as fast as $54 \mathrm{Mbps}$ in the 5-GHz band. 802.11a employs the orthogonal frequency division multiplexing (OFDM) encoding scheme as opposed to either FHSS or DSSS.

- 802.11b: The 802.11 high rate Wi-Fi is an extension to 802.11 that pertains to wireless LANs and yields a connection as fast as $11 \mathrm{Mbps}$ transmission in the $2.4 \mathrm{GHz}$ band. The $802.11 \mathrm{~b}$ specification uses only DSSS. Note that $802.11 \mathrm{~b}$ was actually an amendment to the original 802.11 standard added in 1999 to permit wireless functionality to be analogous to hard-wired Ethernet connections.

- 802.11g: This pertains to wireless LANs and provides $20+$ Mbps in the $2.4 \mathrm{GHz}$ band. 


\subsection{Features Of Wireless LAN (Wi-Fi)}

- Internet Access: A Wi-Fi enabled device such as a personal computer, video game console, Smartphone or digital audio player can connect to the Internet when within range of a wireless network connected to the Internet. The coverage of one or more interconnected access points called hotspots.

- Operational Advantages: Wi-Fi allows the deployment of local area networks (LANs) without wires for client devices, typically reducing the costs of network deployment and expansion. The current version of Wi-Fi Protected Access encryption (WPA2) as of 2010 is considered secure, provided users employ a strong passphrase. New protocols for quality-of-service (WMM) make Wi-Fi more suitable for latency-sensitive applications (such as voice and video) and power saving mechanisms (WMM Power Save) improve battery operation.

\section{DIFFERENCES BETWEEN 3G AND Wi-Fi}

- $3 G$ vs. Wi-Fi - Functioning: As mentioned above, $3 \mathrm{G}$ is a service that is completely provided by the service provider whereas Wi-Fi access can be controlled by a Wi-Fi router located in a specific range from the access point. For availing the Wi-Fi facility, user needs to visit a hotspot which provides a Wi-Fi zone. Today, most malls, cafes, and major streets have Wi-Fi routers for quick Internet access. For availing $3 \mathrm{G}$, you need to get in touch with your service provider.

- $3 G$ vs. Wi-Fi - Range and Signal: The 3G technology scores well over Wi-Fi with regards to range and signal. Since it depends on the mobile service provider, user receives signal reception as long as user is in the network range. In case of Wi$\mathrm{Fi}$, user will be able to receive reception as long as user is within the range of the router situated in the hotspot. Therefore, while traveling, using $3 \mathrm{G}$ access is more appropriate. However, $3 \mathrm{G}$ signals may drop if user is traveling away from metropolitan cities.

- $3 G$ vs. Wi-Fi - Speed: When it comes to the speed of both these technologies, Wi-Fi is faster as compared to $3 \mathrm{G}$. The maximum speed of the ' $\mathrm{N}$ ' standard of the latest Wi-Fi technology is reported to be $600 \mathrm{mbps}$. The data transfer speeds in 3G technology differs according to the kind of device and also whether it is stationary or in motion. The maximum speed on $3 \mathrm{G}$ networks is considered to be about 2.05 mbps. However, if the reception is to be initiated on a cell phone in a moving vehicle, the speed can drop down to $128 \mathrm{kbps}$.

- $\quad 3 G$ vs. Wi-Fi - Cost: The cost of $3 \mathrm{G}$ access depends on the plan chosen from service provider. When it is regarding availing Wi-Fi facilities, user might have to pay the owners of the hotspots. Wi-Fi at some hotspots is free, while others may charge a certain amount. Users of the Wi-Fi facility at hotels and cafes can either pay using a credit card on a payment page hosted by the appropriate authorities, or can pay in cash for getting the network access code. Some hotspots are even code free and user can directly access the web without the access password. 3G consumes more battery than Wi-Fi access.

\section{WIRELESS NETWORK ATTACKS}

The wireless networks consist of four basic components: The transmission of data using radio frequencies; Access points that provide a connection to the organizational network and/or the Client devices (laptops, PDAs, etc.); and Users. Each of these components provides an avenue for attack that can result in the compromise of one or more of the three fundamental security objectives of confidentiality, integrity, and availability.

- Accidental Association: Unauthorized access to company wireless and wired networks can come from a number of different methods and intents. One of these methods is referred to as "accidental association". When a user turns on a computer and it latches on to a wireless access point from a neighboring company's overlapping network, the user may not even know that this has occurred. However, it is a security breach in that proprietary company information is exposed and now there could exist a link from one company to the other. This is especially true if the laptop is also hooked to a wired network.

- Malicious Association: Malicious associations are when wireless devices can be actively made by crackers to connect to a company network through their cracking laptop instead of a company access point (AP). These types of laptops are known as "soft APs" and are created when a cracker runs some software that makes his/her wireless network card look like a legitimate access point.

- Ad-hoc Networks: Ad-hoc networks can pose a security threat. Ad-hoc networks are defined as peer-to-peer networks between wireless computers that do not have an access point in between them. While these types of networks usually have little protection, encryption methods can be used to provide security.

- Non-Traditional Networks: Non-traditional networks such as personal network Bluetooth devices are not safe from cracking and should be regarded as a security risk. Even barcode readers, handheld PDAs, and wireless printers and copiers should be secured. These nontraditional networks can be easily overlooked by IT personnel who have narrowly focused on laptops and access points.

- Identity Theft (MAC Spoofing): Identity theft (or MAC spoofing) occurs when a cracker is able to listen in on network traffic and identify the MAC address of a computer with network privileges. Most wireless systems allow some kind of MAC filtering to only allow authorized computers with specific MAC IDs to gain access and utilize the network. However, a number of programs exist that have network "sniffing" capabilities. Combine these programs with other software that allow a computer to pretend it has any MAC address that the cracker desires and the cracker can easily get around that hurdle.

- Man-in-the-Middle Attacks: A man-in-the-middle attacker entices computers to log into a computer which is set up as a soft AP (Access Point). Once this is done, the hacker connects to a real access point through another wireless card offering a steady flow of traffic through the transparent hacking computer to the real network. The hacker can then sniff the traffic. One type of man-in-the- 
middle attack relies on security faults in challenge and handshake protocols to execute a "deauthentication attack". This attack forces AP connected computers to drop their connections and reconnect with the cracker's soft AP. Man-in-themiddle attacks are enhanced by software such as LANjack and AirJack, which automate multiple steps of the process. What once required some skill can now be done by script kiddies. Hotspots are particularly vulnerable to any attack since there is little to no security on these networks.

- Denial of Service: A Denial-of-Service attack (DoS) occurs when an attacker continually bombards a targeted AP (Access Point) or network with bogus requests, premature successful connection messages, failure messages, and/or other commands. These cause legitimate users to not be able to get on the network and may even cause the network to crash. These attacks rely on the abuse of protocols such as the Extensible Authentication Protocol (EAP).

- Network Injection: In a network injection attack, a cracker can make use of access points that are exposed to non-filtered network traffic, specifically broadcasting network traffic such as "Spanning Tree". The cracker injects bogus networking reconfiguration commands that affect routers, switches, and intelligent hubs. A whole network can be brought down in this manner and require rebooting or even reprogramming of all intelligent networking devices.

- Caffe Latte Attack: The Caffe Latte attack is another way to defeat WEP. It is not necessary for the attacker to be in the area of the network using this exploit. By using a process that targets the Windows wireless stack, it is possible to obtain the WEP key from a remote client. By sending a flood of encrypted ARP requests, the assailant takes advantage of the shared key authentication and the message modification flaws in 802.11 WEP. The attacker uses the ARP responses to obtain the WEP key in less than 6 minutes.

- Interference: Compared to wired systems, wireless networks are frequently subject to electromagnetic interference. This can be caused by other networks or other types of equipment that generate radio waves that are within or close to the radio bands used for communication. Interference can degrade the signal or cause the system to fail.

- Absorption and Reflection: Some materials cause absorption of electromagnetic waves, preventing it from reaching the receiver, in other cases, particularly with metallic or conductive materials reflection occurs. This can cause dead zones where no reception is available. Aluminum foiled thermal isolation in modern homes can easily reduce indoor mobile signals by $10 \mathrm{~dB}$ frequently leading to complaints about bad reception of long distance rural cell signals.

- Multipath Fading: In multipath fading two or more different routes taken by the signal due to reflections can cause the signal to cancel out at certain locations and to be stronger in other places.

- Hidden Node Problem: The hidden node problem occurs in some types of network when a node is visible from a wireless access point (AP) but not from other nodes communicating with that AP. This leads to difficulties in media access control.

Shared Resource Problem: The wireless spectrum is a limited resource and shared by all nodes in the range of its transmitters. Bandwidth allocation becomes complex with multiple participating users. Often users are not aware that advertised numbers (e.g., for IEEE 802.11 equipment or LTE networks) are not their capacity but shared with all other users and thus the individual user rate is far lower.

\section{CONCLUSION}

The 3G and Wi-Fi are both using the wireless technology and protocols for delivering the internet service to the end user. The main difference between $3 \mathrm{G}$ and $\mathrm{Wi}-\mathrm{Fi}$ is that $3 \mathrm{G}$ is the technology which covers large geographical location and provides service over UMTS and HSPA networks while WiFi is suitable for small geographical locations which provide internet service through the Wi-Fi hotspots over wireless access points. Performance of the $3 \mathrm{G}$ and Wi-Fi is changing dynamically at different-different operating environment like large user environment, less user environment, near to base station or access point or long distance from base station or access point. The wireless performance of both the technologies are noticeable but completely depends upon operating environment.

\section{ACKNOWLEDGMENTS}

We would like to acknowledge Prof. H.S. Dhami, Vice Chancellor, Kumaun University, Nainital, Uttarakhand, India and Dr. P.P. Dhayni, Director, G.B. Pant Institute of Himalayan Environment \& Development, Kosi-Katarmal, Almora, Uttarakhand, India for providing adequate resources and continuous support in this research.

\section{REFERENCES}

[1] Wikipedia: https://www.wikipedia.org (explored in October-November, 2014)

[2] Google: https://www.google.co.in (explored in OctoberNovember, 2014)

[3] Wikipedia: http://en.wikipedia.org/wiki (explored in October-November, 2014)

[4] A. Snoeren and H. Balakrishnan (2000). An end-to-end approach to host mobility. Proceedings of the 6th annual international conference on Mobile computing and networking, ACM Press, New York, USA, page 155166.

[5] Birchler, M.; Smyth, P. P.; Martinez, G. and Baker, M. (2003). Future of mobile and wireless communications. BT Technology Journal, vol. 21(3), page 11-21.

[6] Baiamonte, V. and Chiasserini, C.F. (2006). Saving energyduring channel contention in 802.11WLANs. Mobile Networks and Applications, 11(2), page 287-296.

[7] Buellingen, F. and Woerter, M. (2004). Development perspectives, firm strategies and applications in mobile commerce. Journal of Business Research, vo. 57 (12), page 1402-1408.

[8] Buddhikot, M.; Chandranmenon, G.; S. J. Han and Y. W. Lee (2003). Integration of 802.11 and Third Generation Wireless Data Networks. In Proc. IEEE Infocom, April 2003. 
[9] Chevillat, P. R. and Schott, W. (2003). Broadband radio LANs and the evolution of wireless beyond $3 \mathrm{G}$. IBM Journal of Research and Development, vol. 47, page 327-336.

[10] Cardoso, K. V. and Rezende, J. F. (2012). Increasing throughput in dense 802.11 networks by automatic rate adaptation improvement. Wireless Networks, 18(1), page 95-112.

[11] Curwen, P. (2000). Next generation mobile: $2.5 \mathrm{G}$ or $3 \mathrm{G}$ ? Journal of Policy, Regulation and Strategy for Telecommunications, vol. 2 (5), page 455-476.

[12] Dziong, Z.; Khan, F.; Medepalli, K. and Nanda, S. (2002). Wireless Internet access using IS-2000 third generation system: a performance and capacity study. Wireless Networks, vol.8 (4), page. 325-336.

[13] Frodigh, M. (2001). Future generation wireless networks. IEEE Transactions on Personal Communications, vol. 8 (5), page 10-17.

[14] Gerstheimer, O. and Lupp, C (2004). Needs versus technology - the challenge to design third-generation mobile applications. Journal of Business Research, vol. 57 (12), page 1409-1415.

[15] Goodrick, J. and Srivastava, L. (2002). Licensing of third generation (3G) mobile. Journal of Policy, Regulation and Strategy for Telecommunications, vol. 4 (1), page $48-55$

[16] Heikki Karjaluoto (2006). An Investigation of Third Generation (3G) Mobile Technologies and Services. Contemporary Management Research, Vol.2(2), page 91-104.

[17] H. Lin; M. Chatterjee; S. Das and K. Basu (2005). ARC: an integrated admission and rate control framework for competitive wireless CDMA data networks using noncooperative games. IEEE Transactions on Mobile Computing, vol. 4(3), page 243-258.

[18] Kumar, S. (2004). Mobile communications: global trends in the 21st century. International Journal of Mobile Communications, vol. 2 (1), page 67-86.

[19] Lehrer, M. (2004). National lead markets and the design competition for $3 \mathrm{G}$ network applications. Journal of Business Research, vol. 57 (12), page 1397-1401.

[20] Lindemann, C.; Lohmann, M. and Thummler, A. (2003). A unified approach for improving QoS and provider revenue in $3 \mathrm{G}$ mobile networks. Mobile Networks and Applications, vol. 8 (3), page. 209-221.
[21] L. Harju and J. Nurmi (2004). Base-band receiver architecture for UMTS-WLAN interworking applications. Proc. of 9th ISCC, vol. 2, page 678-685.

[22] Nauman Aftab and Ammar Iqbal (2006). 3G Mobile Communication Services Perspective. Master Thesis, Lulea University of Technology, ISSN: 1653-0187.

[23] N. N. Ahmed Hasswa and H. Hassanein (2005). Generic vertical handoff decision function for heterogeneous wireless networks. IFIP Conference on Wireless and Optical Communications, page 239-243.

[24] Nitin Gupta and P.R. Kumar (2004). A Performance analysis of the 802.11 wireless LAN medium access control. Communications in Information System, vol.3 (4), page 279-304.

[25] Park, E. C.; Kim, D.Y.; Cho, C.H.; and So, J. (2007). Improving quality of service and assuring fairness in WLAN access networks. IEEE Transactions on Mobile Computing, 6(4), page 337-350.

[26] Pushpendra Kr. Verma, Preety and Jayant Shekhar (2011). Competition of Wireless Network Access: Wi-Fi Versus 3G. VSRD International Journal of computer science and information technology, Vol. 1(2), page 4452.

[27] Q. Song and A. Jamalipour (2005). Network selection in an integrated wireless LAN and UMTS environment using mathematical modeling and computing techniques. IEEE Wireless Communications, vol. 12(3), page 42-48.

[28] S. Frattasi; H. Fathi; F. Fitzek; R. Prasad and M. Katz (2006). Defining 4G technology from the user's perspective. Network, IEEE, vol. 20(1), page. 35-41.

[29] S. L. Tsao and C. Lin (2002). Design and evaluation of UMTS-WLAN interworking strategies. Proc. of VTC 2002-Fall, page 777-781.

[30] S. Dixit (202). Wireless IP and its challenges for the heterogeneous environment. Wireless Personal Communications, vol. 22(2), page. 261-273.

[31] Tero Ojanpera and Ramjee Prasad (1998). An Overview of Third-Generation Wireless Personal Communications: A European Perspective. IEEE Personal Communications Magazine, vol. 5(6), page 59-65.

[32] Uskela, S. (2003). Key Concepts Evolution Toward Beyond 3G Networks. IEEE WirelessCommunications Magazine, vol. 10(1), page 43-48.

[33] Yuguang Fang (2005). Modeling and Performance Analysis for Wireless Mobile Networks: A New Analytical Approach. IEEE/ACM Transactions on Networking, vol. 13(5), page 989-1002. 